\title{
Tricobezoar gastroduodenal o síndrome de Rapunzel en una niña de 6 años
}

\section{Gastroduodenal trichobezoar or Rapunzel syndrome in a 6-year-old girl}

\author{
Adriana Marcela Arenas-Rojas, ${ }^{*,+}$ Luis Ignacio Villamizar-Durán* \\ * Clínica Materno-Infantil San Luis. Bucaramanga, Santander, Colombia.
}

\begin{abstract}
RESUMEN
Objetivo: Describir el caso de una paciente escolar con síndrome de Rapunzel, dado que se trata de una patología infrecuente en este grupo etario y con pocos casos reportados en Latinoamérica. Reporte de caso: Paciente femenino de seis años con antecedente de tricotilomanía y tricofagia, con dolor abdominal recurrente y desnutrición. En la exploración física se encontró alopecia y masa abdominal indurada y bien delimitada. Por ultrasonido y endoscopía se identificó tricobezoar gastroduodenal de gran tamaño, realizando diagnóstico de síndrome de Rapunzel. Se realizó extracción del tricobezoar mediante laparotomía, y la paciente se mantuvo bajo tratamiento psiquiátrico. Conclusiones: El síndrome de Rapunzel es infrecuente en la edad pediátrica, por lo que requiere alto índice de sospecha para su diagnóstico. El manejo debe ser multidisciplinario.
\end{abstract}

Palabras clave: Síndrome de Rapunzel, tricobezoar, tricotilomanía, tricofagia, escolar.

\section{INTRODUCCIÓN}

El síndrome de Rapunzel corresponde a una forma inusual y extrema de tricobezoar que se localiza desde el estómago, llega a traspasar el píloro y se extiende hasta el intestino delgado y colon derecho. Fue descrito por primera vez en 1969 por el Dr. Vaughan y colaboradores. Su nombre hace alusión al cuento de los hermanos Grimm. En este síndrome ocurre tricofagia (47-50\%) y

\begin{abstract}
Objective: To describe the case of a school-age patient with Rapunzel syndrome, given that it is a rare condition in this age-group and that there are few cases reported in Latin America. Case report: A 6-year-old female patient with a history of trichotillomania and trichophagia, with recurrent abdominal pain and malnutrition. The physical examination revealed alopecia and indurated and well-defined abdominal mass. By ultrasound and endoscopy a large gastroduodenal trichobezoar was found, so the diagnosis of Rapunzel syndrome was made. The trichobezoar was removed by laparotomy, and the patient was subsequently maintained with psychiatric treatment. Conclusions: Rapunzel syndrome is uncommon in pediatric age, requiring a high index of suspicion for its diagnosis. Management must be multidisciplinary.
\end{abstract}

Keywords: Rapunzel syndrome, trichobezoar, trichotillomania, trichophagia, child.

tricotilomanía (33\%) de manera conjunta con trastornos depresivos, de ansiedad y obsesivo-compulsivos..$^{1-4}$

$\mathrm{Su}$ incidencia es rara, revisiones recientes de la literatura revelan alrededor de 100 casos reportados en el mundo. El 95\% de los casos corresponde a mujeres jóvenes, de las cuales el $80 \%$ son menores de 30 años. ${ }^{1-5}$

Se presenta el caso de una paciente con síndrome de Rapunzel, en virtud que su descripción en edad

+Correspondencia: AMAR, adriarenasr@gmail.com

Conflicto de intereses: Los autores declaran que no tienen.

Citar como: Arenas-Rojas AM, Villamizar-Durán LI. Tricobezoar gastroduodenal o síndrome de Rapunzel en una niña de 6 años. Rev Mex Pediatr. 2020; 87(6); 236-239. https://dx.doi.org/10.35366/97687 
pediátrica es inusual y que muy pocos casos han sido reportados en Latinoamérica.

\section{PRESENTACIÓN DEL CASO}

Paciente femenino de seis años procedente de área rural, con evolución clínica de aproximadamente nueve meses, caracterizada por inapetencia y pérdida de peso, asociados con dolor abdominal recurrente. Los padres refieren que previamente se había diagnosticado con anorexia nerviosa, tricotilomanía y tricofagia, por lo cual había recibido manejo por psiquiatría y tratada con fluoxetina durante dos meses.

Al ingreso, se encuentra en regular estado general, con peso $15.9 \mathrm{~kg}$ y estatura $114 \mathrm{~cm}$, con lo cual el IMC es de 12.3, que corresponde -1,2 desviaciones estándar. En la EF se observó una pequeña área de alopecia en región frontoparietal derecha, el abdomen levemente distendido, palpándose una masa indurada, bien delimitada, que ocupaba hipocondrio izquierdo y todo el epigastrio.

En general, los estudios de laboratorio fueron normales, con excepción de hemoglobina $11 \mathrm{~g} / \mathrm{dL}$ y albúmina $2.66 \mathrm{~g} / \mathrm{L}$. En la ecografía abdominal se observó una imagen de apariencia heterogénea e hiperecogénica en hipocondrio izquierdo, redondeada, que generaba sombra acústica posterior, de difícil caracterización y cuantificación dada su extensión. Por lo anterior, se procedió a realizar endoscopía, evidenciando abundante cabello en cuerpo y antro gástrico, que llegaba hasta la segunda porción del duodeno. Con estos hallazgos $\mathrm{y}$, teniendo en cuenta el antecedente psiquiátrico, se confirma el diagnóstico de síndrome de Rapunzel.

Se realizó laparotomía y se encontró tricobezoar de gran tamaño que ocupaba toda la cavidad gástrica con extensión hacia duodeno (Figuras 1 y 2). Se realizó su extracción, transcurriendo la recuperación postquirúrgica sin eventualidades. Al manejo psiquiátrico se agregó risperidona, también se creó un plan de recuperación nutricional. Se dio egreso a la semana de haber realizado el procedimiento quirúrgico. En la actualidad se encuentra en seguimiento ambulatorio, y se ha observado mejoría del apetito, ganancia de peso y adecuada adherencia al tratamiento psiquiátrico.

\section{DISCUSIÓN}

La incidencia del síndrome de Rapunzel es rara en la edad pediátrica y sólo se han reportado algunos casos en el mundo, los cuales predominan en niñas. En 2011 se reportó el caso más joven en el mundo, de un niño de dos años en África. En Colombia, hay algunos reportes de mujeres jóvenes y adolescentes, el más reciente fue en 2017 y se trataba de una adolescente de 14 años. $^{2-6}$

Esta condición parece ser más frecuente en el sexo femenino, ya que, por lo general, tienen el cabello más largo, el cual, al ingerirse tiende a enredarse y formar los tricobezoares. Asimismo la tricotilomanía (deseo irresistible de arrancarse el cabello) es mucho más común en mujeres que en hombres. Se estima que de cinco a $20 \%$ de los casos de tricotilomanía se acompañan de
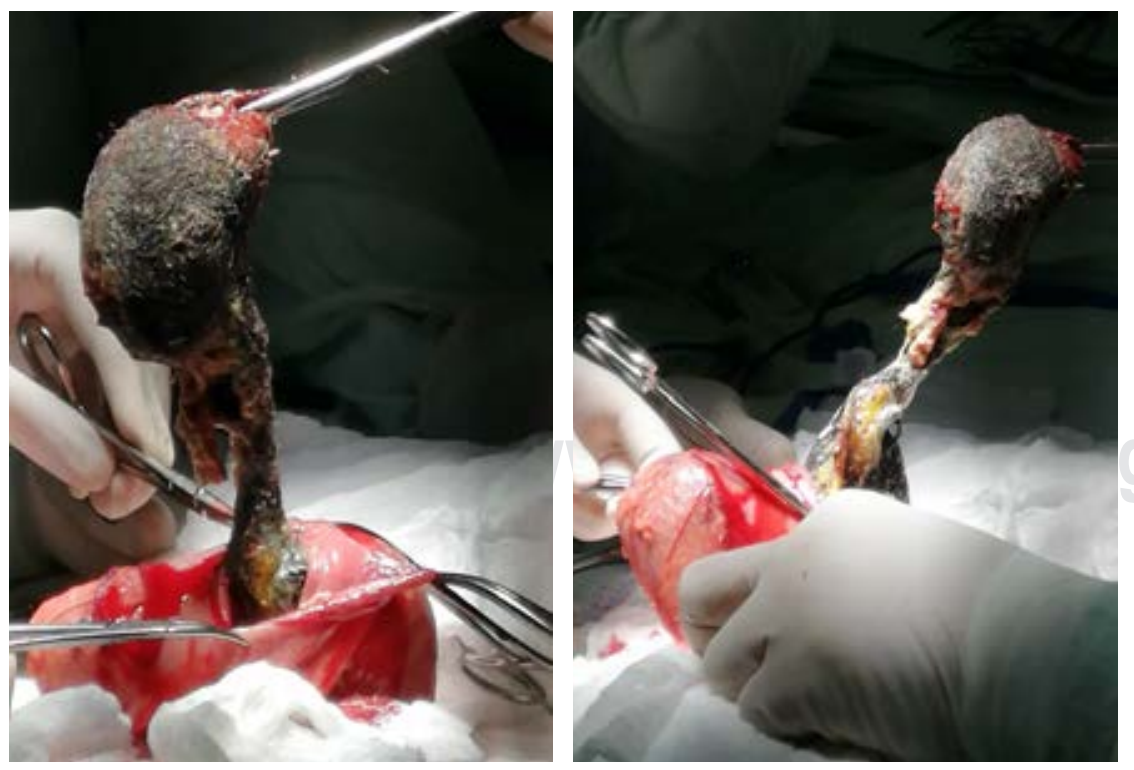

Figura 1:

Extracción quirúrgica del tricobezoar gastroduodenal a través de gastrostomía. 
Figura 2:

Tricobezoar de gran tamaño con
cola que se extendía hasta la
segunda porción del duodeno.
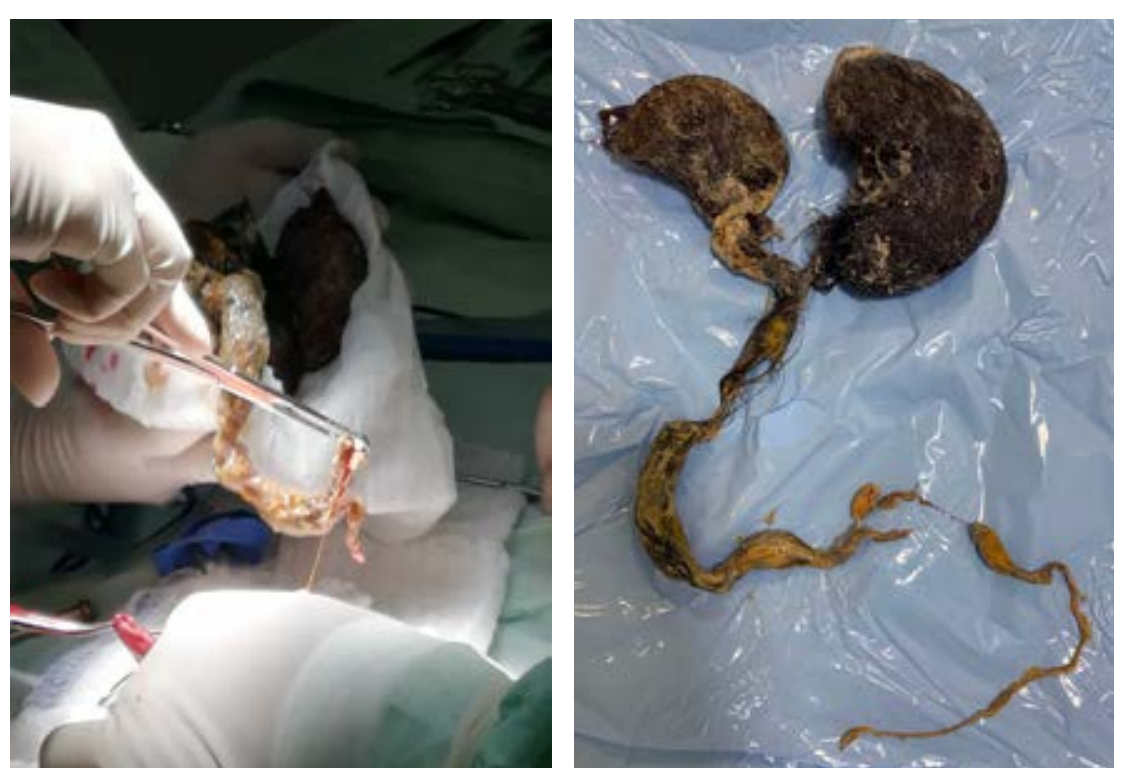

tricofagia. ${ }^{1,4,7}$ La tricotilomanía frecuentemente puede generar zonas de alopecia, lo cual puede ser clave para sospechar el síndrome de Rapunzel..$^{1,5,8-13}$ En nuestra paciente este diagnóstico se realizó puesto que tenía los tres elementos: anorexia nerviosa, tricotilomanía y tricofagia.

Los pacientes con tricobezoares en un principio pueden ser asintomáticos; la sintomatología dependerá de su tamaño, la cual incluye dolor abdominal recurrente (70.2-83\%), masa palpable dura de bordes regulares (signo de Lamerton) (77-87.7\%), náusea y vómito (64.9\%), anorexia, pérdida de peso (38.1\%), plenitud gástrica (11\%), diarrea o constipación (16$32 \%$ ), además de ictericia, hematemesis (6.1\%). Cuando se diagnostica de manera tardía puede asociarse con anemia (62\%), ya sea por malabsorción o por sangrado gastrointestinal. ${ }^{1,2,5}$ En el caso de nuestra paciente, el síntoma cardinal fue el dolor abdominal recurrente asociado con anorexia que llevó a desnutrición.

Las complicaciones asociadas al síndrome de Rapunzel son obstrucción gastrointestinal (80.9\%), perforación (19\%), peritonitis $(5 \%)$, sangrado gastrointestinal, intususcepción (19\%), úlceras gastrointestinales (10\%), obstrucción pancreatobiliar, ictericia obstructiva, pancreatitis, hipoalbuminemia y malabsorción..$^{1-3}$ La perforación gástrica o del intestino delgado, así como la muerte, son complicaciones inusuales que se asocian a negligencia y abandono por parte de los padres o cuidadores. ${ }^{4,11-14}$ En nuestro caso no se evidenciaron complicaciones al momento del diagnóstico.
Para el diagnóstico de síndrome de Rapunzel no es suficiente una radiografía simple de abdomen, ya que, por lo general, sólo se observa una masa poco específica. Las radiografías seriadas de vías digestivas altas podrían mostrar un defecto de llenado del estómago. Mientras que por ecografía puede tener una efectividad diagnóstica de hasta 88\%; los datos son un arco o banda con ecogenicidad aumentada a causa de la mezcla de cabello, aire y comida en el bezoar, acompañado de sombra acústica. Por su parte, la tomografía del abdomen contribuye al diagnóstico hasta en $97 \%$ de los casos, describiendo los tricobezoares como masas heterogéneas y con aire atrapado en su interior; esta característica puede distinguirlos de pseudoquistes, duplicaciones quísticas o tumores. En la endoscopía, observar fragmentos de cabello se considera un hallazgo patognomónico. ${ }^{15-19}$

Los tricobezoares pequeños pueden ser extraídos endoscópicamente; sin embargo, la mayoría de estos son muy grandes. ${ }^{11,19-22}$ Por su parte, la extracción laparoscópica requiere mayor tiempo que la laparotomía convencional, debido a la complejidad del procedimiento. Además, existe el riesgo de derrame del contenido intestinal y del tricobezoar a la cavidad peritoneal. ${ }^{19-23}$ Por lo anterior, el tratamiento de elección para los tricobezoares es la extracción quirúrgica, sobre todo en los de gran tamaño, mediante gastrostomía o enterotomía. En una revisión reciente de 108 casos de tricobezoar, $92.5 \%$ se extrajeron por laparotomía, con una tasa de éxito de $99 \%$ y de complicaciones de $12 \%$. El tiempo promedio de estadía hospitalaria fue de una 
semana. ${ }^{19-23}$ En el caso aquí presentado el tratamiento fue quirúrgico y no se presentaron complicaciones.

Además del manejo quirúrgico, el tratamiento del síndrome de Rapunzel debe ser integral y multidisciplinario, enfocado en manejar la causa subyacente. Se tiene que tomar en cuenta que alrededor del $20 \%$ de los casos pueden ser recidivantes cuando no reciben tratamiento psiquiátrico, puesto que, si no se resuelve la tricotilomanía y la tricofagia, se formarán nuevos tricobezoares. Con base en un manejo integral, el pronóstico a largo plazo parece ser excelente. ${ }^{8,19-23}$

\section{REFERENCIAS}

1. Janssen-Aguilar R, Rochel-Pérez A, Cuevas-Koh OJ, Santos-Zaldivar D, Rodríguez-Cuevas M, Inurreta-Díaz MJ et al. Revisión sistemática del síndrome de Rapunzel. Rev Biomed. 2020; 31(1): c38-57.

2. Ghormallah A, Ghannam AA, Al-Olait Y. Rapunzel syndrome in children. Neonat Pediatr Med. 2006; 1(1): 1-3.

3. Parachuri V, Patel D, Downing M, Kalavakunta J. The youngest case of Rapunzel syndrome: a variation in presentation. Journal of Pediatric Sciences. 2011; 3(3): e90.

4. Castrillón EL, Espinosa MF, Barrios JC, Forero EE. Tricobezoar gastroduodenal en la edad escolar. Caso clínico. Arch Argent Pediatr. 2019; 117(3): e284-e287.

5. Carvajal-Barrios GA, Del Río S, Torres Y. Rapunzel syndrome in a Colombian female adolescent: a case study and literature review. Pediatr. 2017; 50(4): 94-98.

6. Dalshaug GB, Wainer S, Hollaar GL. The Rapunzel syndrome (trichobezoar) causing atypical intussusception in a child: a case report. J Pediatr Surg. 1999; 34: 470-480.

7. Portugal I, Moreira A, Pinto D, Castro M. An unknown abdominal mass in the pediatric population - Rapunzel syndrome. Acta Radiológica Portuguesa. 2019; 31(1): 27-29.

8. Jain M, Solanki SL, Bhatnagar A, Jain PK. An usual case report of Rapunzel syndrome trichobezoar in a 3-year-old boy. Int $J$ Trichology. 2011; 3(2): 102-104.

9. Gupta A, Kaur J, Wadhwa N. Rapunzel syndrome in a seven year old female. Turk J Pediatr. 2017; 59: 598-600.
10. Patel G, Surela R, Patel R. Rapunzel syndrome: surgical problem in a neuropsychiatric disorder. Med J DY Patil Univ. 2015; 8: 813-815.

11. Meier CM, Furtwaengler R. Trichophagia: Rapunzel syndrome in a 7-year-old girl. J Pediatr. 2015; 166(2): 497.

12. Gamal MA, Grover VK, Sameer H. Trichobezoar with gastric ulcer perforation - a case report. Kuwait Med. 2003; 35(4): 296-298.

13. Ventura DE, Mardiros FA, Schettini ST, Delmonte C. Rapunzel syndrome with a fatal outcome in a neglected child. J Pediatr Surg. 2005; 40: 1665-1667.

14. Matejů E, Duchanová S, Kovac P, Moravanský N, Spitz DJ. Fatal case of Rapunzel syndrome in neglected child. Forensic Sci Int. 2009; 190(1-3): e5-e7.

15. Pinilla RO, Vicente ML, González M, Vicente AA, Pinilla ME. Tricobezoar gástrico, revisión de la bibliografía y reporte de un caso. Rev Colomb Cir. 2016; 31: 44-49.

16. Lostra J, Cermeño C, Busquet L, Moguillansky S, Dardanelli E. Causas poco frecuentes de obstrucción en la salida gástrica en Pediatría. Utilidad de la ecografía. Rev Argent Radiol. 2015; 75(1): 32-39.

17. Fallon SC, Slater BJ, Larimer EL, Brandt ML, Lopez ME. The surgical management of Rapunzel syndrome: a case series and literature review. J Pediatr Surg. 2013; 48(4): 830-834.

18. Gorter RR, Kneepkens CM, Mattens EC, Aronson DC, Heij HA. Management of trichobezoar: case report and literature review. Pediatr Surg Int. 2010; 26(5): 457-463.

19. Nirasawa $\mathrm{Y}, \mathrm{Mori} \mathrm{T}$, Ito $\mathrm{Y}$, Tanak H, Seki N, Atomi Y. Laparoscopic removal of a large gastric trichobezoar. J Pediatr Surg. 1998; 33(4): 663-665.

20. Kim JS, Nam CW. A case of Rapunzel syndrome. Pediatr Gastroenterol Hepatol Nutr. 2013; 16(2): V127-V130.

21. Bernal A, González G. Triple tricobezoar. Reporte de un caso. An Med (Mex). 2012; 57(3): 246-251.

22. Lopes LR, Oliveira PS, Pracucho EM, Camargo MA, de Souza Coelho Neto J, Andreollo NA. The Rapunzel syndrome: an unusual trichobezoar presentation. Case Rep Med. 2010; c2010: 841028.

23. Memon SA, Mandhan P, Qureshi JN, Jamil A. Recurrent Rapunzel syndrome - a case report. Med Sci Monit. 2003; 9(9): CS92-CS94. 